\title{
Elevated Myeloperoxidase-DNA Complex Levels in Sera of Patients with IgA Vasculitis
}

\author{
Sora Takeuchi ${ }^{a}$ Tamihiro Kawakami $^{\text {b }}$ Tatsuro Okano $^{\text {a }}$ Haruki Shida $^{c}$ \\ Daigo Nakazawa ${ }^{c}$ Utano Tomaru ${ }^{d}$ Akihiro Ishizu ${ }^{\mathrm{e}}$ Takafumi Kadono $^{\mathrm{a}}$ \\ aDepartment of Dermatology, St. Marianna University School of Medicine, Kawasaki, Japan; b Division of Dermatology, \\ Tohoku Medical and Pharmaceutical University, Sendai, Japan; 'Department of Rheumatology, Endocrinology and \\ Nephrology, Faculty of Medicine and Graduate School of Medicine, Hokkaido University, Sapporo, Japan; dDepartment of \\ Pathology, Faculty of Medicine and Graduate School of Medicine, Hokkaido University, Sapporo, Japan; ${ }^{e}$ Department of \\ Medical Laboratory Science, Faculty of Health Sciences, Hokkaido University, Sapporo, Japan
}

\section{Keywords}

IgA vasculitis · Neutrophil extracellular traps .

Antineutrophil cytoplasmic antibody $\cdot$ Renal complications

\begin{abstract}
Introduction: IgA vasculitis is a systemic disease that results from the entrapment of circulating lgA-containing immune complexes in small-vessel walls in the skin, kidneys, and gastrointestinal tract. An excessive formation of neutrophil extracellular traps (NETs) is involved in the pathogenesis of vasculitis, especially in antineutrophil cytoplasmic antibody (ANCA)-associated vasculitis. This study aimed to clarify whether NETs are implicated in IgA vasculitis. Methods: Twenty-two patients with IgA vasculitis and 4 healthy volunteers were enrolled in this study. Serum levels of myeloperoxidase (MPO)-DNA complex, a fragment derived from NETs, were determined by enzyme-linked immunosorbent assay (ELISA), and the association between MPO-DNA complex levels and clinical parameters was examined. The presence of the ANCA was also assessed by ELISA specific for MPO and proteinase 3 (PR3) and indirect immunofluorescence (IIF), followed by assessing the differences in clinical parameters with and without the ANCA. Results: Serum MPO-DNA com-
\end{abstract}

karger@karger.com www.karger.com/pat

Karger!"

BOPEN ACCESS
(C) 2021 The Author(s)

Published by S. Karger AG, Basel

This is an Open Access article licensed under the Creative Common Attribution-NonCommercial-4.0 International License (CC BY-NC) (http://www.karger.com/Services/OpenAccessLicense), applicable to the online version of the article only. Usage and distribution for commercial purposes requires written permission. plex levels were significantly higher in patients with IgA vasculitis than those in healthy controls. A significant positive correlation between the serum MPO-DNA complex and IgA levels was noted. Interestingly, $63.6 \%$ of IgA vasculitis patients were ANCA-positive in IIF with an atypical pattern, whereas neither MPO-ANCA nor PR3-ANCA was detected by ELISA. These findings indicated that some IgA vasculitis patients possessed the so called minor ANCA. Serum IgA and MPO-DNA complex levels and the frequency of hematuria in the minor ANCA-positive group were significantly higher than in the minor ANCA-negative group. Conclusion: The collective findings suggested that NETs are certainly involved in the pathogenesis of IgA vasculitis.

$$
\begin{aligned}
& \text { (C) } 2021 \text { The Author(s) } \\
& \text { Published by S. Karger AG, Basel }
\end{aligned}
$$

\section{Introduction}

Henoch-Schönlein purpura, IgA vasculitis as adopted by the 2012 Chapel Hill consensus conference (CHCC 2012), is a multisystem disease believed to be a consequence of the entrapment of circulating IgA-containing immune complexes in small-vessel walls in the skin, kidneys, and gastrointestinal tract [1]. 
Neutrophil extracellular traps (NETs) are web-like DNA decorated with antimicrobial proteins, including histones, myeloperoxidase (MPO), and proteinase 3 (PR3), which are released from microorganism-triggered neutrophils [2]. Accumulating evidence indicates that an excessive formation of NETs is involved in the pathogenesis of vasculitis, especially in antineutrophil cytoplasmic antibody (ANCA)-associated vasculitis [3, 4]. In ANCAassociated vasculitis, the ANCA binds with MPO and PR3, expressed on neutrophils activated by pro-inflammatory stimuli; consequently, excessive activation of neutrophils occurs. These neutrophils release reactive oxygen species, proteolytic enzymes, and NETs, injuring the endothelium of small vessels.

An excessive formation of NETs induces microinflammation in the small-vessel wall via immunothrombus formation [5, 6]. In contrast, Aleyd et al. [7] have demonstrated that IgA immune complexes in the plasma and synovial fluid of patients with rheumatoid arthritis induced NETs. Based on this evidence, it is hypothesized that NETs are implicated in the pathogenesis of IgA vasculitis.

To verify the hypothesis, 22 patients with IgA vasculitis and 4 healthy volunteers were enrolled in this study. Serum levels of the MPO-DNA complex, a fragment derived from NETs, were determined by enzyme-linked immunosorbent assay (ELISA), and the association between MPO-DNA complex levels and clinical parameters was examined. The presence of the ANCA was also assessed by ELISA specific for MPO and PR3 and indirect immunofluorescence (IIF), followed by assessing the differences in clinical parameters with and without the ANCA.

\section{Patients and Methods}

\section{Patients}

This study reviewed the sequential records of 22 patients with IgA vasculitis seen at the Department of Dermatology, St. Marianna University School of Medicine between 2010 and 2016. Patients presented with initial cutaneous manifestations characteristic of IgA vasculitis, with palpable purpura over the lower extremities. Based on the presence of leukocytoclastic vasculitis, vascular IgA depositions, and the absence of thrombocytopenia or known hematologic or connective tissue disorders, they were diagnosed with IgA vasculitis. The diagnosis was made according to the criteria defined by the American College of Rheumatology [8] and the KAWAKAMI algorithm [9]. None of the patients demonstrated any coexisting malignancy, other autoimmune diseases, or viral hepatitis and was positive for mixed cryoglobulinemia.
Table 1. Clinical and serological findings in IgA vasculitis

\begin{tabular}{ll}
\hline Characteristic & $(n=22)$ \\
\hline Age, years & $38.90 \pm 19.25$ \\
Gender (M/F) & $8 / 14$ \\
Arthralgia, $n(\%)$ & $11(50.0)$ \\
Abdominal pain, $n(\%)$ & $8(36.4)$ \\
Proteinuria, $n(\%)$ & $14(63.6)$ \\
Hematuria, $n(\%)$ & $15(68.2)$ \\
CRP, mg/dL & $1.62 \pm 2.33$ (normal, $<0.3)$ \\
IgA, mg/dL & $340.7 \pm 175.6$ (normal, 95-360) \\
ANCA in IIF, $n(\%)$ & $14(63.6)$ \\
\hline
\end{tabular}

ANCA, antineutrophil cytoplasmic antibody; CRP, C-reactive protein; IIF, indirect immunofluorescence.

\section{Serum Samples}

All blood samples were immediately centrifuged at $1,500 \mathrm{~g}$ for 30 min at $4^{\circ} \mathrm{C}$. Serum samples were collected and stored at $-80^{\circ} \mathrm{C}$ until analysis. Immunological assessments were performed on sera collected when each patient presented with palpable purpura over the lower extremities. For controls, 4 healthy volunteers were included.

\section{Detection of MPO-ANCA, PR3-ANCA, C-Reactive Protein,} and $\operatorname{IgA}$

Serum MPO-ANCA and PR3-ANCA were determined by ELISA (Medical \& Biological Laboratories, Tokyo, Japan) according to the manufacturer's protocol. Serum IgA and C-reactive protein (CRP) levels were assayed using laser nephelometry (Nihon Kohden, Tokyo, Japan).

\section{IIF for ANCA}

A commercially available IIF kit (Medical \& Biological Laboratories) was used for ANCA detection according to the manufacturer's instructions. In brief, ANCA detection by IIF was performed on ethanol-fixed and formalin-fixed neutrophils. Serum samples were diluted in phosphate-buffered saline and screened at a dilution of 1:20. Interpretation of immunofluorescence results was based on the characteristics of the ethanol- and formalin-fixed specimens.

\section{Quantification of MPO-DNA Complex}

The amount of in vivo NET formation can be monitored as MPODNA complexes in the serum [3]. ELISA for MPO-DNA complexes was carried out, as described previously $[10,11]$. In brief, microtiter plates (Thermo Fisher Scientific, Waltham, MA, USA) were first coated with the monoclonal antihuman MPO antibody $(5 \mu \mathrm{g} / \mathrm{mL}$; AbD Serotec, Kidlington, UK) and incubated overnight at $4^{\circ} \mathrm{C}$. After blocking with $1 \%$ bovine serum albumin, the sera (final dilution at 1:3) and horseradish peroxidase-conjugated anti-DNA antibody in the Cell Death Detection kit (Roche Diagnostics, Tokyo, Japan) were applied to the wells, followed by color development according to the manufacturer's instructions. The serum from a patient with MPOANCA-associated vasculitis served as a positive control.

Statistical Analysis

Differences in patient parameters were assessed using the Mann-Whitney U-test and $\chi^{2}$ test for independent samples. Cor- 

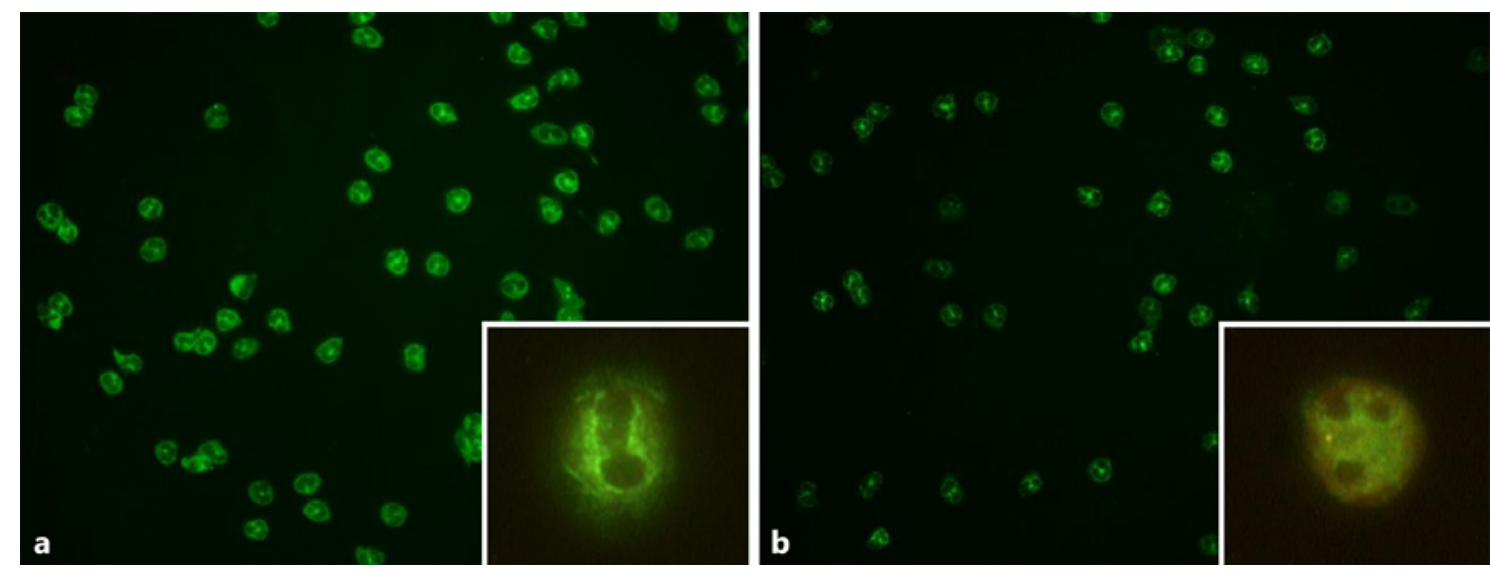

Fig. 1. IIF detection of ANCA in IgA vasculitis. IIF demonstrated an atypical pattern with fine rim-like staining of the nuclear periphery in ethanol-fixed neutrophils (a) and a cytoplasmic fluorescence in formalin-fixed neutrophils (b). insets High-power field of view. IIF, indirect immunofluorescence; ANCA, antineutrophil cytoplasmic antibody.

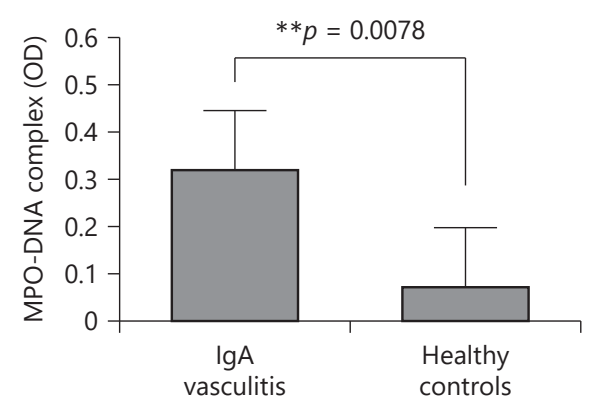

a

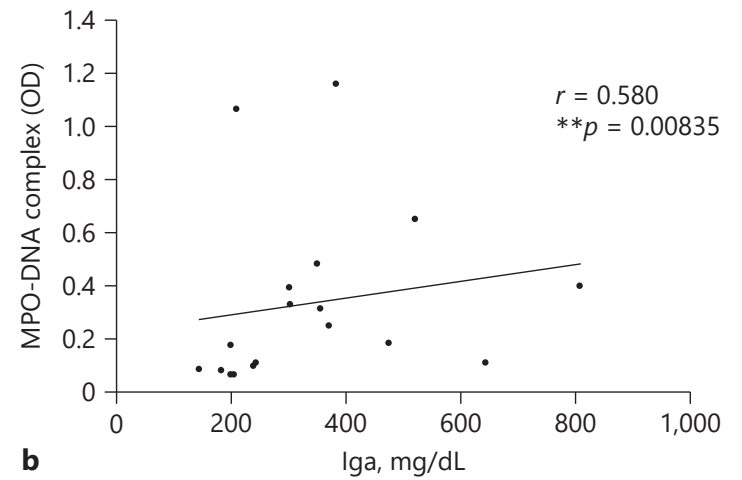

b

Fig. 2. Correlation between the serum MPO-DNA complex and IgA levels in IgA vasculitis. a Serum MPO-DNA complex levels were significantly higher in IgA vasculitis patients than in healthy controls. b A significant positive correlation was noted between the serum MPO-DNA complex and IgA levels in IgA vasculitis patients. MPO, myeloperoxidase.

relations between quantitative parameters were measured by Spearman's rank correlation coefficient. A $p$ value $<0.05$ was considered statistically significant. All data are expressed as mean \pm standard deviation.

\section{Results}

\section{Clinical and Serological Findings in Patients with IgA} Vasculitis

The clinical and serological features of $22 \operatorname{IgA}$ vasculitis patients are listed in Table 1. Fourteen (63.6\%) patients were positive for serum CRP. Serum MPO-ANCA and PR3-ANCA were negative according to ELISA in all patients. In contrast, the ANCA was detected in 14 (63.6\%) patients based on IIF. When ethanol-fixed neutrophils were subjected to IIF, positive fluorescence was detected as fine rim-like signals of the nuclear periphery distinct from the typical perinuclear-ANCA pattern (Fig. 1a), whereas the typical cytoplasmic-ANCA pattern was observed on formalin-fixed neutrophils (Fig. 1b). ANCAs that demonstrate such characteristics are atypical ANCAs [12]. All sera from ANCA-positive IgA vasculitis patients enrolled in this study demonstrated the atypical ANCA 

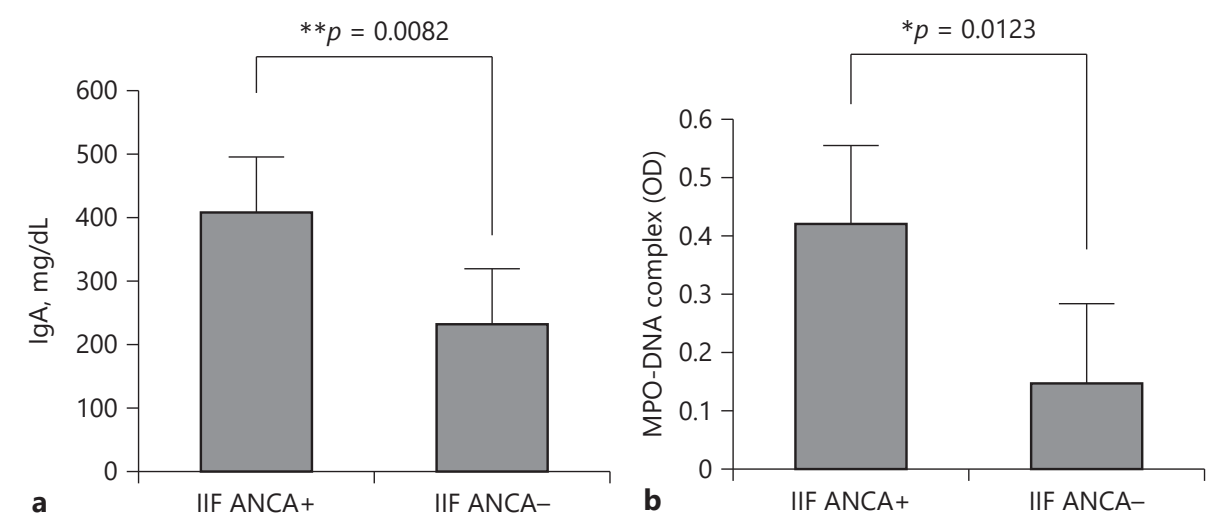

Fig. 3. Differences in serum IgA and MPO-DNA complex levels in IgA vasculitis based on ANCA production. a Comparison of serum IgA levels between the minor ANCA-positive and ANCA-negative groups. b Comparison of serum MPO-DNA complex levels between the minor ANCA-positive and ANCA-negative groups. MPO, myeloperoxidase; ANCA, antineutrophil cytoplasmic antibody; IIF, indirect immunofluorescence.

pattern. It has been shown that ANCAs recognize neutrophil cytoplasmic antigens other than MPO and PR3, namely, minor ANCAs, which usually exhibit the atypical pattern [12]. These findings indicated that some IgA vasculitis patients possessed the so called minor ANCA.

\section{Correlation between Serum MPO-DNA Complex and}

IgA Levels in IgA Vasculitis

Circulating MPO-DNA complex levels in the serum of 22 IgA vasculitis patients and 4 healthy volunteers were determined by ELISA. Serum MPO-DNA complex levels were significantly higher in IgA vasculitis patients than healthy controls (Fig. 2a). A significant positive correlation between the serum MPO-DNA complex and IgA levels in 22 IgA vasculitis patients was noted (Fig. 2b; $r=$ $0.580, p=0.00835$ ).

\section{Differences in Serum IgA and MPO-DNA Complex Levels in IgA Vasculitis Patients Based on ANCA Production}

Twenty-two IgA vasculitis patients were divided into 2 subgroups: minor ANCA-positive and ANCA-negative groups. Both serum IgA and MPO-DNA complex levels in the minor ANCA-positive group were significantly higher than those in the minor ANCA-negative group (Fig. 3a, b). In addition, the frequency of hematuria in the minor ANCA-positive group was significantly higher than that in the minor ANCA-negative group $(p=0.0195)$.
Similar trends were noted in the frequency of proteinuria, although the difference did not reach statistical significance $(p=0.0540)$. Any correlation among clinical and laboratory parameters, such as serum CRP levels, arthralgia, and abdominal pain, between the minor ANCA-positive and minor ANCA-negative groups was not observed.

\section{Discussion}

This study demonstrated that serum levels of the MPO-DNA complex, a fragment derived from NETs, were significantly higher in IgA vasculitis patients than in healthy controls. A significant positive correlation was noted between the serum MPO-DNA complex and IgA levels in IgA vasculitis patients. It is generally accepted that increased serum IgA concentration is a characteristic laboratory finding during the acute stage of $\operatorname{IgA}$ vasculitis [13-15]. Thus, it is suggested that NETs may be associated with the disease activity of IgA vasculitis.

The 2 major categories of small-vessel vasculitis are ANCA-associated vasculitis and immune complex vasculitis, both of which often affect small vessels in the skin [16]. Interestingly, minor ANCA that recognized neutrophil cytoplasmic antigens other than MPO and PR3 was detected in $63.6 \%$ of IgA vasculitis patients in this study. These findings suggested the presence of a common im- 
munological basis underlying ANCA-associated vasculitis and immune complex vasculitis, including IgA vasculitis, which can explain similar manifestations in the skin, rather than the complication of the 2 diseases.

Serum IgA and MPO-DNA complex levels and the frequency of hematuria in the minor ANCA-positive group were significantly higher than those in the minor ANCAnegative group. Similar trends were noted in the frequency of proteinuria between the groups. The collective findings suggested the association between minor ANCA production and disease activity of IgA vasculitis, including renal manifestations.

It is debatable whether the elevated NET formation is a result or cause of IgA vasculitis. IgA immune complexes have been demonstrated to induce NETs [7]. MPOANCA and PR3-ANCA can perpetuate a vicious circle of NET production that maintains the delivery of antigenchromatin complexes to the immune system [3]. Similarly, some minor ANCAs can enhance NET formation $[17,18]$. Although further studies are needed to identify antigens recognized by minor ANCAs in IgA vasculitis patients and determine whether the minor ANCA induces NETs, this study suggested that NETs are certainly involved in the pathogenesis of $\operatorname{Ig}$ A vasculitis.

\section{Acknowledgment}

This work was supported by the Scientific Research Fund of the Ministry of Education, Science, Sports and Culture, Japan (Grantin-Aid for Scientific Research nos. 13670902, 15591199, 17591187, and 19591331).

\section{Statement of Ethics}

This study was approved by the St. Marianna University Ethics Committee (No. 3755), and written informed consent was obtained from all patients and healthy volunteers.

\section{Conflict of Interest Statement}

The authors have no conflicts of interest to declare.

\section{Funding Sources}

No funding was received for this publication.

\section{Author Contributions}

Sora Takeuchi, Tamihiro Kawakami, and Akihiro Ishizu designed the research. Sora Takeuchi, Tatsuro Okano, Haruki Shida, and Daigo Nakazawa performed the experiments. Sora Takeuchi, Tatsuro Okano, Haruki Shida, Daigo Nakazawa, Utano Tomaru, and Akihiro Ishizu analyzed the data. Sora Takeuchi, Tamihiro Kawakami, and Akihiro Ishizu wrote the manuscript. Takafumi Kadono supervised this study. All the authors discussed the results and contributed to the preparation of the manuscript.

\section{Data Availability Statement}

All data generated or analyzed during this study are included in this article. Further inquiries can be directed to the corresponding author.

\section{References}

1 Jennette JC, Falk RJ, Bacon PA, Basu N, Cid MC, Ferrario F, et al. 2012 revised international chapel hill consensus conference nomenclature of vasculitides. Arthritis Rheum. 2013;65(1):1-11.

2 Brinkmann V, Reichard U, Goosmann C, Fauler B, Uhlemann Y, Weiss DS, et al. Neutrophil extracellular traps kill bacteria. Science. 2004;303(5663):1532-5.

3 Kessenbrock K, Krumbholz M, Schönermarck U, Back W, Gross WL, Werb Z, et al. Netting neutrophils in autoimmune smallvessel vasculitis. Nat Med. 2009;15(6):623-5.

4 Nakazawa D, Masuda S, Tomaru U, Ishizu A. Pathogenesis and therapeutic interventions for ANCA-associated vasculitis. Nat Rev Rheumatol. 2019;15(2):91-101.

5 Doring Y, Weber C, Soehnlein O. Footprints of neutrophil extracellular traps as predictors of cardiovascular risk. Arterioscler Thromb Vasc Biol. 2013;33(8):1735-6.
6 Nakazawa D, Ishizu A. Immunothrombosis in severe COVID-19. EBioMedicine. 2020;59: 102942.

7 Aleyd E, Al M, Tuk CW, van der Laken CJ, van Egmond M. IgA Complexes in plasma and synovial fluid of patients with rheumatoid arthritis induce neutrophil extracellular traps via FcaRI. J Immunol. 2016;197(12): 4552-9.

8 Mills JA, Michel BA, Bloch DA, Calabrese LH, Hunder GG, Arend WP, et al. The American college of rheumatology 1990 criteria for the classification of Henoch-Schönlein purpura. Arthritis Rheum. 1990;33(8): 1114-21.

9 Kawakami T. New algorithm (KAWAKAMI algorithm) to diagnose primary cutaneous vasculitis. J Dermatol. 2010;37(2):11324.

10 Miyoshi A, Yamada M, Shida H, Nakazawa D, Kusunoki Y, Nakamura A, et al. Circulat- ing neutrophil extracellular trap levels in well-controlled type 2 diabetes and pathway involved in their formation induced by high-dose glucose. Pathobiol. 2016;83(5): 243-51.

11 Morita S, Nakamaru Y, Nakazawa D, Hattanda F, Shida H, Kusunoki Y, et al. Elevated level of myeloperoxidase-deoxyribonucleic acid complex in the middle ear fluid obtained from patients with otitis media associated with antineutrophil cytoplasmic antibody-associated vasculitis. Otol Neurotol. 2018;39(4):e257e62.

12 Savige JA, Davies DJ, Gatenby PA. Anti-neutrophil cytoplasmic antibodies (ANCA): their detection and significance: report from workshops. Pathology. 1994;26(2):186-93.

13 Kawakami T, Watabe H, Mizoguchi M, Soma Y. Elevated serum IgA anticardiolipin antibody levels in adult Henoch-Schönlein purpura. Br J Dermatol. 2006;155(5):983-7. 
14 Kawakami T, Yamazaki M, Mizoguchi M, Soma Y. High titer of serum antiphospholipid antibody levels in adult Henoch-Schönlein purpura and cutaneous leukocytoclastic angiitis. Arthritis Rheum. 2008;59(4):561-7.

15 Kawakami T, Takeuchi S, Arimura Y, Soma Y. Elevated antilysosomal-associated membrane protein-2 antibody levels in patients with adult Henoch-Schönlein purpura. Br J Dermatol. 2012;166(6):1206-12.
16 Sunderkotter $\mathrm{CH}$, Zelger B, Chen KR, Requena L, Piette W, Carlson JA, et al. Nomenclature of cutaneous vasculitis: dermatologic addendum to the 2012 revised international chapel hill consensus conference nomenclature of vasculitides. Arthritis Rheumatol. 2018;70(2):171-84.

17 Shida H, Nakazawa D, Tateyama Y, Miyoshi A, Kusunoki Y, Hattanda F, et al. The presence of anti-lactoferrin antibodies in a sub- group of eosinophilic granulomatosis with polyangiitis patients and their possible contribution to enhancement of neutrophil extracellular trap formation. Front Immunol. 2016;7:636.

18 Takeda S, Watanabe-Kusunoki K, Nakazawa D, Kusunoki Y, Nishio S, Atsumi T. The Pathogenicity of BPI-ANCA in a patient with systemic vasculitis. Front Immunol. 2019;10: 1334. 